\author{
Suwen Chen ${ }^{1,2}$, Guoyi Tang ${ }^{1}$, Shenhua Song ${ }^{2}$, \\ Guolin Song ${ }^{1}$, Victor Gromov ${ }^{3}$
}

\author{
${ }^{1}$ Advanced Materials Institute, Graduate School at Shenzhen, Tsinghua University (China) \\ ${ }^{2}$ Department of Materials Science and Engineering, Shenzhen Graduate School, Harbin Institute of Technology (China) \\ ${ }^{3}$ Siberian State University of Industry (Russia)
}

\title{
EFFECT OF ELECTROPULSING TREATMENT ON THE MECHANICAL PROPERTIES OF Q235 STEEL STRIP
}

\begin{abstract}
The elctropulsing treatment (EPT) has been successfully applied to the processing of a low carbon Q235 steel strip. Comparing with the conventional heat treatment (CHT), a proper EPT is capable of achieving the similar or even better effect on the mechanical properties of the steel strip. It was found that the optimum combination of the EPT parameters are $180 \mathrm{~V}$ in voltage and $500 \mathrm{~Hz}$ in frequency $(180 \mathrm{~V}-500 \mathrm{~Hz})$ leading to a combination of tensile strength-elongation of $371 \mathrm{MPa}-47,5 \%$. Optical microscopy analyses indicates that the EPT/180 V - 500 Hz for Q235 steel strip can accelerate the formation of the completely recrystallized microstructure in which the grain size become relatively finer and more uniform compared to the elongated one formed in the cold-rolled sample. Such phenomenon is consistent with the improvement of the mechanical performance of the Q235 steel sample under the EPT. However, the EPT with inadequate frequency can only result in partial recrystallization of the grains, while the one with an exceed frequency may lead to the apparent grain growth within the sample. Both cases can not produce satisfactory combination of strength and ductility for the steel samples.
\end{abstract}

Keywords: electropulsing treatment, the conventional heat treatment, mechanical properties, microstructure.

Electropulsing treatment (EPT) was firstly discoved to reduce the resistance of deformation within the metals in the 1969 [1]. EPT is an instantaneous high energy input method, which nowadays has been widely used to improve the microstructure and mechanical properties of materials. Studies by Wang and his co-workers [2, 3] indicate that the improvement of plasticity of the TA15 sheet with EPT can be realized by the EPT-induced local recrystallization and damage healing. Conrad et al. [4, 5] reported that EPT may refine the grains and raise the nucleation rate of recrystallization during annealing of the cold worked copper. Such phenomena are attributed to the EPT-enhanced mobility of dislocations [6]. Zhu and Lee [7 - 9] found that the tensile test under the EPT may produce a much higher elongation for a $\mathrm{Zn}-\mathrm{Al}$ alloy compared to the conventional tensile test. And the relationship between the elongation and the electropulsing can be explained by dislocation dynamics and microstructural evolution. A study by Liu et al. [10] indicates that the EPT can increase the shape memory effect of a Fe-based shape memory alloy due to accelerating the precipitation of $\mathrm{NbC}$ carbide particles. Recently, Xu et al. [11 - 13] have obtained ultrafine recrystallized grains in a cold rolled AZ31 strip by using EPT, and the behavior of recrystallization during the EPT may be due to the acceleration of dislocation propagation and annihilation as well as the enhancement of vacancy mobility. Overall, the EPT is very likely to improve the plasticity of the metals or alloys without apparently losing its strength.

Low carbon steel Q235 is widely used as an engineering structural material. It has the room-temperature yield strength of approximately $235 \mathrm{MPa}$ with an excellent ductility, i.e. it possesses sound comprehensive mechanical properties. However, in the production of Q235 steel strip, the steel has to undergo a cold rolling process, which normally leads to work-hardening. Therefore, annealing is required right after the cold rolling process. The conventional heat treatment (CHT) of the steel in a furnace usually takes a long time interval and therefore a large quantity of electrical energy is consumed. In order to reduce the energy consumption and improve the mechanical properties of the steel strip, the EPT was successfully employed in the present work to treat a cold-rolled Q235 steel strip.

\section{Experimental}

The material used was a cold-rolled Q235 steel strip which was commercially produced with a width of $20 \mathrm{~mm}$ and a thickness of $1 \mathrm{~mm}$. The chemical composition of the steel (wt. \%) is $0,098 \mathrm{C}, 0,38 \mathrm{Mn}, 0,12 \mathrm{Si}, 0,027 \mathrm{~S}, 0,002 \mathrm{P}$ and $0,025 \mathrm{Cr}$ with a balance of Fe. Both EPT and CHT for the steel strip samples were carried out for comparative studies.

The procedures of a typical EPT are schematically illustrated in Fig. 1. The Q235 strip specimen was on-line treated by a continuous electropulsing where the strip was

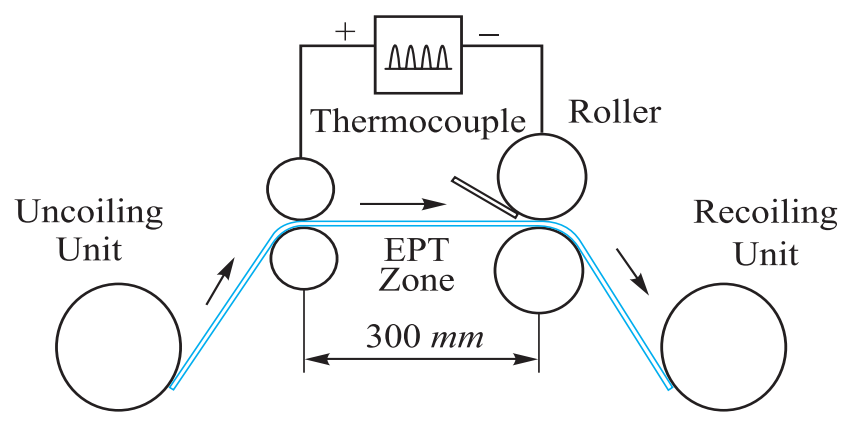

Fig. 1. Schematic illustration of the EPT process 
moving at a constant speed of $14 \mathrm{~m} / \mathrm{min}$ through a distance of $300 \mathrm{~mm}$ between the two electrodes. During the process, it took about $21 \mathrm{~s}$ for the strip to move from the anode to the cathode. A self-made electropulsing generator was utilized to create the multiple positive electropulses. The pressure between the electrode rollers and the strip was kept at a sufficient level to maintain a good electrical contact without causing any undesirable deformation of the strip. Electropulses having the duration of about $70 \mu$ s were applied on the strip specimens at various voltages and frequencies. The current parameters including frequency, root-mean-square (RMS) current, amplitude and duration of current pulses were constantly monitored and recorded by using a Halleffect sensor connected to an oscilloscope. The temperature of the Q235 strip specimen was measured by using a Raytek MX2 infrared thermoscope. In parallel, the CHT were also carried out for the same Q235 strip sample in a furnace at $873 \mathrm{~K}$ for about $2 \mathrm{~h}$ for comparisons purpose. Both the EPT and the CHT were performed under a natural air environment. Various parameters for both EPT and CHT are listed in Table.

The strip specimen surfaces after both EPT and CHT were well polished and etched with a solution of $4 \%$ nitric acid and $96 \%$ ethanol, and observed by using an optical microscopy. Tensile tests for the strip specimens with a gauge size of $100 \times 20 \times 1 \mathrm{~mm}$ were performed on a universal testing machine at a cross-head speed of $5 \mathrm{~mm} / \mathrm{min}$.

\section{Results and discussion}

It can be noticed by the parameters of EPT shown in Table, the temperature of the specimen under EPT increases with the increasing of frequency at the same voltage, or with the increasing of voltage at the same frequency. A higher frequency or voltage leads to a larger RMS value of current density and more powerful thermal and athermal effects [14].

The values of the tensile strength and elongation for the cold-rolled specimens with the CHT and the EPT are also given in Table. In the cold-rolled state, the tensile strength of the sample was $728 \mathrm{MPa}$ while its elongation was merely $3,4 \%$. With the CHT, the steel sample had the tensile strength reduced to $361 \mathrm{MPa}$ while an elongation increased to $44,5 \%$. With the EPT, at the same frequency of $500 \mathrm{~Hz}$ the tensile strength varies from $658 \mathrm{MPa}$ to $371 \mathrm{MPa}$ to $376 \mathrm{MPa}$ to $540 \mathrm{MPa}$ as the inputed voltage alters from $150 \mathrm{~V}$ to $180 \mathrm{~V}$ to $200 \mathrm{~V}$ to $220 \mathrm{~V}$ with the elongation changing from $6,9 \%$ to $47,5 \%$ to $34,5 \%$ to $11,9 \%$. Therefore, the optimum combination of strength and ductility of Q235 steel strip sample was achieved by using EPT at a voltage of $180 \mathrm{~V}$. Under such circumstances, both the strength and ductility (i.e. $371 \mathrm{MPa}$ and $47,5 \%$ ) of the steel specimens are higher than those achieved by using the CHT (i.e. $361 \mathrm{MPa}$ and 44,5\%). Although the differences in the property values between the steel specimens with the EPT and the CHT are not quite remarkable, the EPT still posses the obvious advantage of a large amount of electrical energy and time savings compared to the CHT. At the same voltage of $180 \mathrm{~V}$, both the strength and the ductility of the treated steel specimens vary with the frequencies, and the best results of these properties were obtained at the frequency of $500 \mathrm{~Hz}$. Hence, the optimum electrical parameter combination of voltage and frequency of the EPT for Q235 steel strip is $180 \mathrm{~V} / 500 \mathrm{~Hz}$.

The microstructures of the specimens subjected to different treatments are shown in Fig. 2. it can be obviously seen from Fig. 2, $a$ that the cold-rolled specimen shows an elongated microstructure. After the CHT, the elongated microstructure of the steel evolved to a recrystallized one in which the grain size is quite small and uniform (see Fig. 2, b). After the EPT at various parameters, the elongated microstructure of the steel evolved to different ones. For the sample treated by the EPT/ $180 \mathrm{~V}-300 \mathrm{~Hz}$, only a small amount of elongated microstructure were recrystallized (see Fig. 2, c). It is likely due to the temperature of the specimen $(460 \mathrm{~K})$ during the EPT is too low to initiate recrystallization. The microstructure of the specimen obtained by using the EPT/ $180 \mathrm{~V}-500 \mathrm{~Hz}$ is similar to that

\section{The parameters of EPT and CHT for the Q235 steel strip}

\begin{tabular}{c|c|c|c|c|c|c|c}
\hline Sample & $U, \mathrm{~V}$ & $f, \mathrm{~Hz}$ & $J_{m}, \mathrm{~A} / \mathrm{mm}^{2}$ & $J_{e}, \mathrm{~A} / \mathrm{mm}^{2}$ & $T, \mathrm{~K}$ & $\sigma_{\mathrm{p}}, \mathrm{MPa}$ & $e, \%$ \\
\hline ACR & - & - & - & - & 298 & 728 & 3,4 \\
\hline CHT & - & - & - & - & 873 & 361 & 44,5 \\
\hline EPT 1 & 150 & 500 & 121 & 10,26 & 533 & 658 & 6,9 \\
\hline EPT 2 & 180 & 300 & 114 & 12,57 & 460 & 665 & 6,5 \\
\hline EPT 3 & 180 & 500 & 107 & 14,57 & 674 & 371 & 47,5 \\
\hline EPT 4 & 180 & 700 & 114 & 16,76 & 883 & 426 & 22,6 \\
\hline EPT 5 & 200 & 500 & 130 & 17,33 & 823 & 376 & 34,5 \\
\hline EPT 6 & 220 & 500 & 137 & 18,00 & 903 & 540 & 11,9 \\
\hline
\end{tabular}

N o t e. ACR stands for as cold-rolled; $J_{m}$ is the amplitude of current-density of electropulsing; and $J_{e}$ represents the RMS value of the current during EPT and is related to the Joule heating effect; TS stands for the tensile strength; and EL stands for the elongation. 

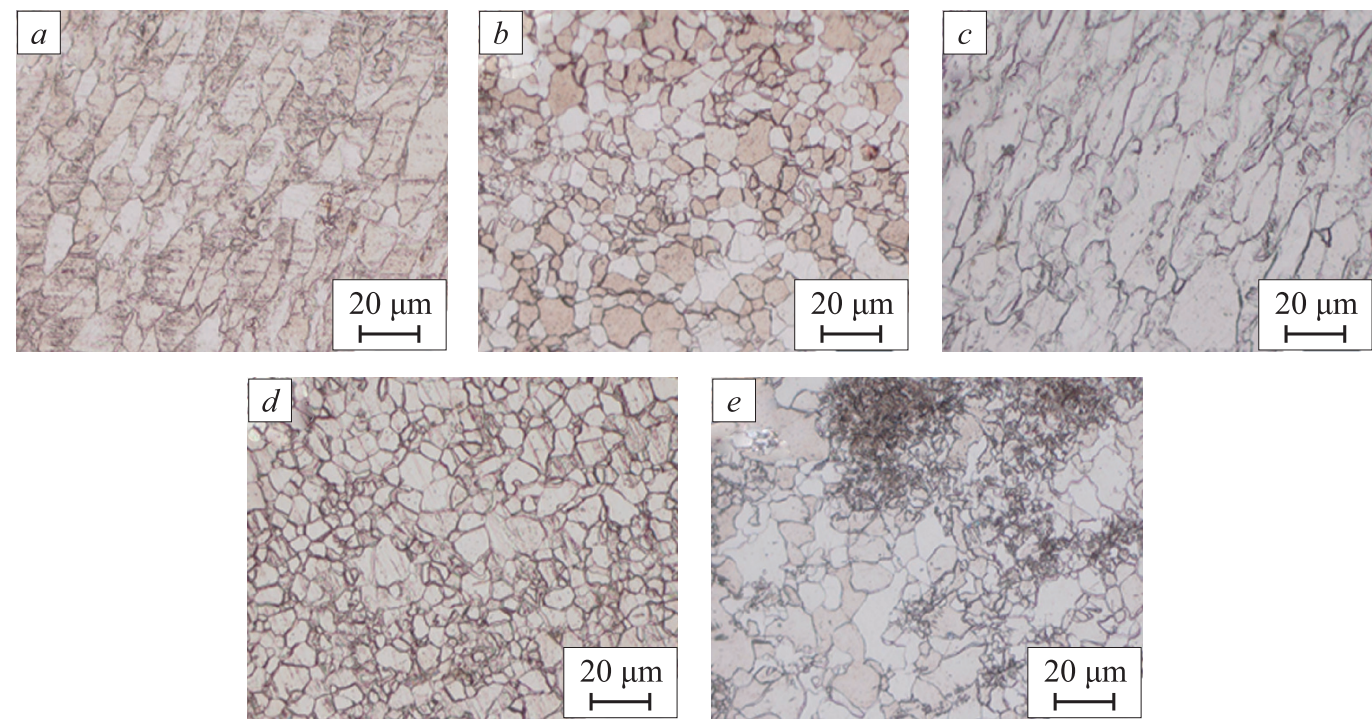

Fig. 2. Microstructures of the samples subjected to different treatments:

$a$ - cold rolling; $b-\mathrm{CHT} ; c-\mathrm{EPT} / 180 \mathrm{~V}-300 \mathrm{~Hz} ; d-\mathrm{EPT} / 180 \mathrm{~V}-500 \mathrm{~Hz} ; e-\mathrm{EPT} / 180 \mathrm{~V}-700 \mathrm{~Hz}$

obtained by using the CHT. In this case, the temperature of the sample is $674 \mathrm{~K}$ which was nearly 200 degree lower than that used in the CHT (see Table). This implies that the EPT can considerably promote the kinetic process of recrystallization. After the EPT/180 V $-700 \mathrm{~Hz}$, the grain growth of the steel took place and its microstructure also became less uniform. This is probably because the EPTinduced temperature $(883 \mathrm{~K})$ was too high for a proper recrystallization, and led to a worse combination of strength and ductility.

Since recrystallization can be explained by motion and interaction of dislocations, an increased mobility of the dislocations produced by the electropulsing may be able to promote recrystallization. Many studies [15 - 17] have indicated that the relationship between electrons and dislocations consists of thermal and athermal effects when electropulsing is applied on metals. However, the real mechanism of athermal effects is still not very clear. One possible explanation is that it results from an additional force which the periodic impulse electrons exert on a dislocation, i.e. a so-called "electron wind" [4]. It is supposed that the electropulsing can effectively accelerate the migration of vacancies, which is related to the dislocation climb. The dislocation climb has a close relationship with the total flux of the diffusing atoms $J$ as given by Eq. (1) [18]:

$$
J=J_{t h}+J_{a t h}=J_{t h}+\frac{D_{1}}{k T}\left(K_{e w} \Omega J_{m}+N_{1} \rho e Z_{1}^{*} J_{m}\right)
$$

where $J_{t h}$ and $J_{a t h}$ are the fluxes of the diffusing atoms due to the thermal and athermal effects, $D_{1}$ is the lattice diffusing coefficient, $k$ is the Boltzmann constant, $\Omega$ is the atom volume, $N_{1}$ is the number of lattice atoms per unit volume, $T$ is the absolute temperature, $K_{e w}$ is the coefficient of electron wind force, and $e Z_{1}^{*}$ is the effective charges of lattice atoms.
In addition, the number of dislocations climbing into the subgrain boundaries, in terms of nc, per unit time can be given as Eq. (2). [19]:

$$
\frac{d n_{c}}{d t}=J \frac{\Omega}{b},
$$

where $b$ is the Burgers Vector.

It can be clearly found from Eq. (1) that even though the flux of the diffusing atoms due to thermal effect in the electropulsing forming process is small at the relatively low temperature, it is possible to enhance $J$ remarkably by increasing $J_{m}$. Therefore, the climbing of dislocations in subgrain boundaries per unit time can speed up according to Eq.(2).

The detailed mechanism of athermal effect may be deduced as the following viewpoint. It is known that electron movement is ranging between $K_{0}$ and $K_{\max }$ periodically when electropulsing is applied to the metals. $K_{\max }$ increases with $J_{m}$ as $K_{\max }=m^{*} v_{\max }=m^{*} J_{m} / e n$, where $m^{*}$ is the effective electron mass, vmax is the maximum electron velocity, $e$ represents the electron charge and $\mathrm{n}$ is the electron density or the number of electrons per unit volume. According to the impulse law, the varying momentum can transfer to the impacting force as $K_{\max }=\int_{0}^{\tau} F d t$, where $F$ is the impacting force and $\tau$ is the electropulsing duration. Due to a very short duration $(70 \mu \mathrm{s})$ in these experiments, the electron impacting force may become tremendous, which furthermore accelerates atoms irregular movement based on the initial thermal effect (i.e., the joule heat effect). Certainly, the higher the $J_{m}$ is imported, the more superior athermal effect will be produced as $F$ increases sharply.

The coupling of thermal effect and athermal effect exists in the EPT for Q235 steel strip. However, it should be 
noted that the fraction of athermal effect contributing to plastic deformation needs further studying.

\section{Conclusions}

The low carbon Q235 steel strip has been successfully processed by using the elctropulsing treatment technique. There is an optimum combination of the EPT parameters which can lead to the best combination of strength and ductility of the sample. In the present case, it was found that the optimum combination of the EPT parameters are $180 \mathrm{~V}$ in voltage and $500 \mathrm{~Hz}$ in frequency $(180 \mathrm{~V}-500 \mathrm{~Hz})$ leading to a combination of tensile strength-elongation of $371 \mathrm{MPa}-47,5 \%$, which is better than that achieved by using the conventional heat treatment. After the EPT, the microstructure in the sample is a fully recrystallized one in which the grain size is very fine and uniform. Such phenomenon is responsible for the improvement of the mechanical performance (i.e. the best combination of strength and ductility) of the Q235 steel sample under the EPT.

\section{REFERENCES}

1. Troitskii O.A. // Journal of Experimental and Theoretical Physics. 1969. Vol. 10. P. $18-22$.

2. Wang Z., S ong H. // Journal of Alloys and Compounds. 2009. Vol. 470. P. $522-530$.

3. Wang Z., S ong H. // Transactions of Nonferrous Metals Society of China. 2009. Vol. 19. P. $409-413$.

4. Conrad H., Karam N., Mannan S. // Scripta Materialia. 1983. Vol. 17. P. $411-416$.
5. Conrad H., Karam N., Mannan S. // Scripta Materialia. 1984. Vol. 18. P. $275-280$.

6. Elektrostimulirovannaya ductility of metals and alloys / V.E. Gromov, L.B. Zuev, E.V. Kozlov, V.Y. Tsellermaer. - Moscow: Nedra, 1996. -290 .

7. Zhu Y.H., Le e W.B., Li u X.M. et al. // Materials Science and Engineering A. 2009. Vol. 501. P. 125 - 132.

8. To S., Zhu Y.H., L e e W.B. et al.// Materials Transaction. 2009. Vol. 50. P. $1105-1112$.

9. To S., Z hu Y.H., L e e W.B. et al.// Materials Transaction. 2009. Vol. 50. P. $2772-2777$.

10. Li u W.B., Wen Y.H., Li N., Yang S.Z. // Journal of Alloys and Compounds. 2009. Vol. 472. P. 591 - 594.

11. $\mathrm{Xu}$ Z.H., Tang G.Y., D ing F. et al.// Applied Physics A. 2007. Vol. 88. P. $429-433$.

12. Guan L., Tang G.Y., Ji ang Y.B., Chu P.K. // Journal of Alloys and Compounds. 2009. Vol. 487. P. $309-313$.

13. Guan L., Tang G.Y., $\mathrm{Chu}$ P.K., Jiang Y.B. // Journal of Materials Research. 2009. Vol. 24. P. $3674-3679$.

14. Ji ang Y.B., Tang G.Y., Guan L. et al. // Journal of Materials Research. 2008. Vol. 23. P. $2685-2690$.

15. Troitskii O.A., Spitsyn V.I., Sokolov N.V., Ryzhk ov V.G. // Physica Status Solidi A. 1979. Vol. 52. P. $2685-2690$.

16. Sprecher A.F., Mamnnan S.L., Conrad H. // Acta Materialia. 1986. Vol. 34. P. $1145-1150$.

17. Okazaki K., Kagawa M., Conard H.// Materials Science and Engineering A. 1980. Vol. 45. P. $109-116$.

18. Sprecher F., Mannan S.L., Conrad H.// Acta Materialia. 1983. Vol. 17. P. $769-772$.

19. $\mathrm{Xu}$ Z.H., Tang G.Y., Ti an S.Q. et al. // Journal of Materials Research. 2007. Vol. 182. P. 128 - 133.

(C) 2013 Suwen Chen, Guoyi Tang, Shenhua Song, Guolin Song, Victor Gromov Received December 12, 2012. 